\title{
THE KERNEL OF THE MAGNUS REPRESENTATION OF THE AUTOMORPHISM GROUP OF A FREE GROUP IS NOT FINITELY GENERATED
}

\author{
Takao Satoh \\ Department of Mathematics, Graduate School of Science, Kyoto University, \\ Kitashirakawaoiwake-cho, Sakyo-ku, Kyoto city 606-8502, Japan

\begin{abstract}
In this paper, we show that the abelianization of the kernel of the Magnus representation of the automorphism group of a free group is not finitely generated.
\end{abstract}

\section{INTRODUCTION}

Let $F_{n}$ be a free group of rank $n \geq 2$, and Aut $F_{n}$ the automorphism group of $F_{n}$. Let denote $\rho$ : Aut $F_{n} \rightarrow$ Aut $H$ the natural homomorphism induced from the abelianization $F_{n} \rightarrow H$. The kernel of $\rho$ is called the IA-automorphism group of $F_{n}$, denoted by $\mathrm{IA}_{n}$. The subgroup $\mathrm{IA}_{n}$ reflects many richness and complexity of the structure of Aut $F_{n}$, and plays important roles on various studies of Aut $F_{n}$.

Although the study of the IA-automorphism group has a long history since its finitely many generators were obtained by Magnus [14] in 1935, the combinatorial group structure of $\mathrm{IA}_{n}$ is still quite complicated. For instance, any presentation for $\mathrm{IA}_{n}$ is not known in general. Nielsen [19] showed that $\mathrm{IA}_{2}$ coincides with the inner automorphism group, hence, is a free group of rank 2 . For $n \geq 3$, however, $\mathrm{IA}_{n}$ is much larger than the inner automorphism group Inn $F_{n}$. Krstić and McCool [11] showed that $\mathrm{IA}_{3}$ is not finitely presentable. For $n \geq 4$, it is not known whether $\mathrm{IA}_{n}$ is finitely presentable or not.

In general, one of the most standard ways to study a group is to consider its representations. If a representation of the group is given, it is important to determine whether it is faithful or not. Furthermore, if not, it is also worth studying how far it is from faithful, namely, to determine its kernel. In this paper, we consider these matters for the Magnus representation

$$
r_{M}: \mathrm{IA}_{n} \rightarrow \mathrm{GL}(n, \mathbf{Z}[H])
$$

of $\mathrm{IA}_{n}$. (See Subsection 2.3.)

Classically, the Magnus representation was used to study certain subgroups of $\mathrm{IA}_{n}$. One of the most famous subgroup is the pure braid group $P_{n}$. The restriction of $r_{M}$ to $P_{n}$ is called the Gassner representation. (For a basic materials for the braid group and the pure braid group, see [4] for example.) It is known due to Magnus and Pelso [15] that $\left.r_{M}\right|_{P_{n}}$ is faithful for $n=3$. Although the faithfulness of $\left.r_{M}\right|_{P_{n}}$ has been studied

2000 Mathematics Subject Classification. 20F28(Primary), 20J06(Secondly).

Key words and phrases. Magnus representation, Johnson homomorphism, IA-automorphism group of a free group.

${ }^{1}$ e-address: takao@math.kyoto-u.ac.jp 
for a long time by many authors, it seems, however, still open problem to determine it for $n \geq 4$.

Another important subgroup is the Torelli $\mathcal{I}_{g, 1}$ subgroup of the mapping class group of a surface. By a classical work of Dehn and Nielsen, the Torelli group $\mathcal{I}_{g, 1}$ is considered as a subgroup of $\mathrm{IA}_{2 g}$. Suzuki [22] showed that the restriction $\left.r_{M}\right|_{\mathcal{I}_{g, 1}}$ to $\mathcal{I}_{g, 1}$ is not faithful for $g \geq 2$. Furthermore, by a recent remarkable work of Church and Farb [7], it is known that the abelianization of the kernel of $\left.r_{M}\right|_{\mathcal{I}_{g, 1}}$ is not finitely generated for $g \geq 2$.

From the facts as mentioned above, it is immediately seen that $r_{M}$ itself is not faithful. There are, however, few results for the study of the kernel of $r_{M}$. Let $\mathcal{K}_{n}$ be the kernel of $r_{M}$. In our previous paper [21], we showed that the abelianization $\mathcal{K}_{n}^{\mathrm{ab}}$ of $\mathcal{K}_{n}$ contains a certain free abelian group of finite rank. In this paper, we show that $r_{M}$ is far from faithful. That is,

Theorem 1. (= Theorem 4.1 and Proposition [5.2.) For $n \geq 2, \mathcal{K}_{n}^{\mathrm{ab}}$ is not finitely generated.

In order to prove the theorem for $n \geq 3$, we consider embeddings of $\mathcal{K}_{n}$ into the IA-automorphism group of a free group of higher rank. Then, taking advantage of the first Johnson homomorphisms, we detect infinitely many linearly independent elements in $\mathcal{K}_{n}^{\mathrm{ab}}$. For $n=2$, in particular, we show

Proposition 1. $\mathcal{K}_{2}=\left[\left[F_{2}, F_{2}\right],\left[F_{2}, F_{2}\right]\right]$

by using a usual and classical argument in the combinatorial group theory.

This paper consists of six sections. In Section 2, we recall the IA-automorphism group and the Magnus representation of the automorphism group of a free group. In Section 3, we prove the main theorem for $n \geq 3$. In Section 5, we consider the case where $n=2$.

\section{Contents}

1. Introduction

2. Preliminaries

2.1. Notation and conventions

2.2. IA-automorphism group

2.3. Magnus representation

3. Embeddings of the IA-automorphism group

3.1. Subgroups $W_{n, d}$ of $F_{n}$

3.2. Action of $\mathrm{IA}_{n}$ on $W_{n, d}$

4. Lower bounds on $H_{1}\left(\mathcal{K}_{n}, \mathbf{Z}\right)$

5. The case where $n=2$

6. Acknowledgments

References 


\section{Preliminaries}

In this section, after fixing notation and conventions, we recall the IA-automorphism group and the Magnus representation of Aut $F_{n}$.

\subsection{Notation and conventions.}

Throughout the paper, we use the following notation and conventions. Let $G$ be a group and $N$ a normal subgroup of $G$.

- The abelianization of $G$ is denoted by $G^{\text {ab }}$.

- For any group $G$, we denote by $\mathbf{Z}[G]$ the integral group ring of $G$.

- The group Aut $G$ of $G$ acts on $G$ from the right. For any $\sigma \in$ Aut $G$ and $x \in G$, the action of $\sigma$ on $x$ is denoted by $x^{\sigma}$.

- For an element $g \in G$, we also denote the coset class of $g$ by $g \in G / N$ if there is no confusion.

- For elements $x$ and $y$ of $G$, the commutator bracket $[x, y]$ of $x$ and $y$ is defined to be $[x, y]:=x y x^{-1} y^{-1}$.

\subsection{IA-automorphism group.}

In this paper, we fix a basis $x_{1}, \ldots, x_{n}$ of $F_{n}$. Let $H:=F_{n}^{\mathrm{ab}}$ be the abelianization of $F_{n}$ and $\rho:$ Aut $F_{n} \rightarrow$ Aut $H$ the natural homomorphism induced from the abelianization of $F_{n}$. In the following, we identify Aut $H$ with the general linear group $\operatorname{GL}(n, \mathbf{Z})$ by fixing the basis of $H$ induced from the basis $x_{1}, \ldots, x_{n}$ of $F_{n}$. The kernel $\mathrm{IA}_{n}$ of $\rho$ is called the IA-automorphism group of $F_{n}$. It is clear that the inner automorphism group $\operatorname{Inn} F_{n}$ of $F_{n}$ is contained in $\mathrm{IA}_{n}$. In general, however, $\mathrm{IA}_{n}$ for $n \geq 3$ is much larger than Inn $F_{n}$. In fact, Magnus [14] showed that for any $n \geq 3, \mathrm{IA}_{n}$ is finitely generated by automorphisms

$$
K_{i j}: x_{t} \mapsto \begin{cases}x_{j}{ }^{-1} x_{i} x_{j}, & t=i, \\ x_{t}, & t \neq i\end{cases}
$$

for distinct $i, j \in\{1,2, \ldots, n\}$ and

$$
K_{i j l}: x_{t} \mapsto \begin{cases}x_{i}\left[x_{j}, x_{l}\right], & t=i, \\ x_{t}, & t \neq i\end{cases}
$$

for distinct $i, j, l \in\{1,2, \ldots, n\}$ such that $j<l$. In this paper, for the convenience, we also consider an automorphism $K_{i j l}$ for $j \geq l$ defined as above. We remark that $K_{i j j}=1$ and $K_{i l j}=K_{i j l}^{-1}$.

Recently, Cohen-Pakianathan [5, 6] CFarb [8] and Kawazumi [10] independently showed

$$
\mathrm{IA}_{n}^{\mathrm{ab}} \cong H^{*} \otimes_{\mathbf{Z}} \Lambda^{2} H
$$

as a $\operatorname{GL}(n, \mathbf{Z})$-module where $H^{*}:=\operatorname{Hom}_{\mathbf{Z}}(H, \mathbf{Z})$ is the $\mathbf{Z}$-linear dual group of $H$. This isomorphism is induced from the first Johnson homomorphism

$$
\tau_{1}: \mathrm{IA}_{n} \rightarrow H^{*} \otimes_{\mathbf{z}} \Lambda^{2} H
$$

(For a basic materials for the Johnson homomorphisms, see [16], Kawazumi [10] and 20] for example.) Then we see that (the coset classes of) the Magnus generators $K_{i j}$ s and $K_{i j k}$ s form a basis of $\mathrm{IA}_{n}^{\mathrm{ab}}$ as a free abelian group. 
In Section 4, we use the following lemmas.

Lemma 2.1. For $n \geq 4$, and distinct integers $1 \leq i, j, l, m \leq n$, let $\omega \in \mathrm{IA}_{n}$ be an automorphism defined by

$$
x_{t} \mapsto \begin{cases}x_{i} x_{j} x_{l}^{-1} x_{m} x_{j}^{-1} x_{l} x_{m}^{-1}, & t=i, \\ x_{t}, & t \neq i .\end{cases}
$$

Then, we have

$$
\omega=K_{i l} K_{i m l} K_{i j m} K_{i l j} K_{i l}^{-1} \in \mathrm{IA}_{n}
$$

and in particular,

$$
\omega=K_{i m l}+K_{i j m}+K_{i l j} \in \mathrm{IA}_{n}^{\mathrm{ab}} .
$$

Proof. Since $K_{i l} K_{i m l} K_{i j m} K_{i l j} K_{i l}^{-1}$ fix $x_{t}$ for $t \neq i$, it suffices to consider the image of $x_{i}$. Then we see

$$
\begin{aligned}
x_{i} & \stackrel{K_{i l}}{\longrightarrow} x_{l}^{-1} x_{i} x_{l} \stackrel{K_{i m l}}{\longrightarrow} x_{l}^{-1} x_{i}\left[x_{m}, x_{l}\right] x_{l}=x_{l}^{-1} x_{i} x_{m} x_{l} x_{m}^{-1} \stackrel{K_{i j m}}{\longrightarrow} x_{l}^{-1} x_{i}\left[x_{j}, x_{m}\right] x_{m} x_{l} x_{m}^{-1} \\
& =x_{l}^{-1} x_{i} x_{j} x_{m} x_{j}^{-1} x_{l} x_{m}^{-1} \stackrel{K_{i l j}}{\longrightarrow} x_{l}^{-1} x_{i}\left[x_{l}, x_{j}\right] x_{j} x_{m} x_{j}^{-1} x_{l} x_{m}^{-1} \\
& =x_{l}^{-1} x_{i} x_{l} x_{j} x_{l}^{-1} x_{m} x_{j}^{-1} x_{l} x_{m}^{-1} \stackrel{K_{i l}^{-1}}{\longrightarrow} x_{i} x_{j} x_{l}^{-1} x_{m} x_{j}^{-1} x_{l} x_{m}^{-1} .
\end{aligned}
$$

This completes the proof of Lemma 2.1.

Similarly, we obtain the following lemmas. Since the proofs are done by a straightforward calculation as above, we leave them to the reader as exercises.

Lemma 2.2. For $n \geq 5$, and distinct integers $1 \leq i, j, l, m, p \leq n$, let $\omega \in \mathrm{IA}_{n}$ be an automorphism defined by

$$
x_{t} \mapsto \begin{cases}x_{i} x_{j} x_{l}^{-1} x_{p} x_{m} x_{p}^{-1} x_{j}^{-1} x_{l} x_{p} x_{m}^{-1} x_{p}^{-1}, & t=i, \\ x_{t}, & t \neq i .\end{cases}
$$

Then, we have

$$
\omega=K_{m p} K_{i l} K_{i m l} K_{i j m} K_{i l j} K_{i l}^{-1} K_{m p}^{-1} \in \mathrm{IA}_{n}
$$

and in particular,

$$
\omega=K_{i m l}+K_{i j m}+K_{i l j} \in \mathrm{IA}_{n}^{\mathrm{ab}} .
$$

Lemma 2.3. For $n \geq 5$, and distinct integers $1 \leq i, j, l, m, p \leq n$, let $\omega \in \mathrm{IA}_{n}$ be an automorphism defined by

$$
x_{t} \mapsto \begin{cases}x_{i} x_{p} x_{j} x_{p}^{-1} x_{l}^{-1} x_{p} x_{m} x_{p}^{-1} x_{p} x_{j}^{-1} x_{p}^{-1} x_{l} x_{p} x_{m}^{-1} x_{p}^{-1}, & t=i, \\ x_{t}, & t \neq i .\end{cases}
$$

Then, we have

$$
\omega=K_{j p} K_{m p} K_{i l} K_{i m l} K_{i j m} K_{i l j} K_{i l}^{-1} K_{m p}^{-1} K_{j p}^{-1} \in \mathrm{IA}_{n},
$$

and in particular,

$$
\omega=K_{i m l}+K_{i j m}+K_{i l j} \in \mathrm{IA}_{n}^{\mathrm{ab}} .
$$




\subsection{Magnus representation.}

In this subsection we recall the Magnus representation of Aut $F_{n}$. (For details, see [4].) For each $1 \leq i \leq n$, let

$$
\frac{\partial}{\partial x_{i}}: \mathbf{Z}\left[F_{n}\right] \rightarrow \mathbf{Z}\left[F_{n}\right]
$$

be the Fox derivation defined by

$$
\frac{\partial}{\partial x_{i}}(w)=\sum_{j=1}^{r} \epsilon_{j} \delta_{\mu_{j}, i} x_{\mu_{1}}^{\epsilon_{1}} \cdots x_{\mu_{j}}^{\frac{1}{2}\left(\epsilon_{j}-1\right)} \in \mathbf{Z}\left[F_{n}\right]
$$

for any reduced word $w=x_{\mu_{1}}^{\epsilon_{1}} \cdots x_{\mu_{r}}^{\epsilon_{r}} \in F_{n}, \epsilon_{j}= \pm 1$. Let $\mathfrak{a}: F_{n} \rightarrow H$ be the abelianization of $F_{n}$. We also denote by $\mathfrak{a}$ the ring homomorphism $\mathbf{Z}\left[F_{n}\right] \rightarrow \mathbf{Z}[H]$ induced from $\mathfrak{a}$. For any matrix $A=\left(a_{i j}\right) \in \operatorname{GL}\left(n, \mathbf{Z}\left[F_{n}\right]\right)$, set $A^{\mathfrak{a}}=\left(a_{i j}^{\mathfrak{a}}\right) \in \operatorname{GL}(n, \mathbf{Z}[H])$. The Magnus representation $r_{M}$ : Aut $F_{n} \rightarrow \mathrm{GL}(n, \mathbf{Z}[H])$ of Aut $F_{n}$ is defined by

$$
\sigma \mapsto\left(\frac{\partial x_{i}{ }^{\sigma}}{\partial x_{j}}\right)^{\mathfrak{a}}
$$

for any $\sigma \in \operatorname{Aut} F_{n}$. This map is not a homomorphism but a crossed homomorphism. Namely,

$$
r_{M}(\sigma \tau)=r_{M}(\sigma)^{\tau^{*}} \cdot r_{M}(\tau)
$$

where $r_{M}(\sigma)^{\tau^{*}}$ denotes the matrix obtained from $r_{M}(\sigma)$ by applying the automorphism $\tau^{*}: \mathbf{Z}[H] \rightarrow \mathbf{Z}[H]$ induced from $\rho(\tau) \in \operatorname{Aut}(H)$ on each entry. Hence by restricting $r_{M}$ to $\mathrm{IA}_{n}$, we obtain a homomorphism $\mathrm{IA}_{n} \rightarrow \mathrm{GL}(n, \mathbf{Z}[H])$, also denote it by $r_{M}$.

Let $\mathcal{K}_{n}$ be the kernel of the homomorphism $r_{M}: \mathrm{IA}_{n} \rightarrow \mathrm{GL}(n, \mathbf{Z}[H])$. The main purpose of the paper is to show that the abelianization $\mathcal{K}_{n}^{\mathrm{ab}}$ of $\mathcal{K}_{n}$ is not finitely generated. More precisely, we prove that it contains a free abelian group of infinite rank.

Here we consider the group $\mathcal{K}_{n}$ from the view point of the metabelianization of $F_{n}$. Let $F_{n}^{M}$ be the quotient group of $F_{n}$ by the second derived subgroup $\left[\left[F_{n}, F_{n}\right],\left[F_{n}, F_{n}\right]\right]$ of $F_{n}$. The group $F_{n}^{M}$ is called the free metabelian group of rank $n$. The abelianization of $F_{n}^{M}$ is canonically isomorphic to $H=F_{n}^{\mathrm{ab}}$. Let $\mu_{n}$ : Aut $F_{n} \rightarrow$ Aut $F_{n}^{M}$ be the induced homomorphism from the action of Aut $F_{n}$ on $F_{n}^{M}$. It is known that $\mu_{n}$ is surjective for $n \geq 2$ except for $n=3$. (See [1] for $n=2$, and see [3] for $n \geq 4$.) Let $\mathrm{IA}_{n}^{M}$ be the IA-automorphism group of $F_{n}^{M}$. Namely, the group $\mathrm{IA}_{n}^{M}$ consists of automorphisms of $F_{n}^{M}$ which act on the abelianization of $F_{n}^{M}$ trivially. Then the restriction of $\mu_{n}$ induces a homomorphism $\mathrm{IA}_{n} \rightarrow \mathrm{IA}_{n}^{M}$, also denoted by $\mu_{n}$.

Now, in [1], Bachmuth constructed a faithful representation

$$
r_{M}^{\prime}: \mathrm{IA}_{n}^{M} \rightarrow \mathrm{GL}(n, \mathbf{Z}[H])
$$

using the Magnus representation of $F_{n}^{M}$. Then we can easily see that $r_{M}=r_{M}^{\prime} \circ \mu_{n}$ by observing the image of the Magnus generators of $\mathrm{IA}_{n}$. (For details, see [1].) Therefore, the faithfulness of $r_{M}^{\prime}$ induces $\mathcal{K}_{n}=\operatorname{Ker}\left(\mu_{n}\right)$. In particular, we have

$$
\mathcal{K}_{n}=\left\{\sigma \in \operatorname{Aut} F_{n} \mid x^{-1} x^{\sigma} \in\left[\left[F_{n}, F_{n}\right],\left[F_{n}, F_{n}\right]\right], x \in F_{n}\right\} \subset \mathrm{IA}_{n} .
$$




\section{Embeddings of The IA-AUTOMORPhism GROUP}

In this section, we consider certain finitely generated subgroups of the free group $F_{n}$, and its automorphism group.

\subsection{Subgroups $W_{n, d}$ of $F_{n}$.}

For any integer $d \geq 2$, let $C_{d}$ be the cyclic group of order $d$ generated by $s$. Let $\iota_{n, d}: F_{n} \rightarrow C_{d}$ be a homomorphism defined by

$$
x_{i}^{\iota_{n, d}}:= \begin{cases}s, & i=1, \\ 1, & i \neq 1 .\end{cases}
$$

We denote by $W_{n, d}$ the kernel of $\iota_{n, d}$. Then, $W_{n, d}$ is also a free group, and its rank is given by $d(n-1)+1$ since the index of $W_{n, d}$ in $F_{n}$ is $d$. Furthermore, applying the Reidemeister-Schreier method to the following data:

$$
\begin{aligned}
X & :=\left\{x_{1}, x_{2}, \ldots, x_{n}\right\} ; \text { the set of generators of } F_{n}, \\
T & :=\left\{1, x_{1}, \ldots, x_{1}^{d-1}\right\} ; \text { a Schreier-transversal of } W_{n, d} \text { of } F_{n},
\end{aligned}
$$

we see that a set

$$
\left\{(t, x) \in W_{n, d} \mid t \in T, \quad x \in X, \quad(t, x) \neq 1\right\}
$$

form a basis of $W_{n, d}$, where $(t, x)=t x(\overline{t x})^{-1}$ and a map ${ }^{-}: F_{n} \rightarrow T$ is defined by the condition that

$$
W_{n, d} y=W_{n, d} \bar{y} \subset F_{n}
$$

for any $y \in F_{n}$. (For details for the Reidemeister-Schreier method, see [12] for example.)

Then the set (2) is written down as

$$
\begin{aligned}
& x_{1}^{d}, \\
& x_{2}, x_{1} x_{2} x_{1}^{-1}, \ldots, x_{1}^{d-1} x_{2} x_{1}^{-(d-1)}, \\
& \vdots \\
& x_{n}, x_{1} x_{n} x_{1}^{-1}, \ldots, x_{1}^{d-1} x_{n} x_{1}^{-(d-1)} .
\end{aligned}
$$

In the following, we fit this set as a basis of $W_{n, d}$.

\subsection{Action of $\mathrm{IA}_{n}$ on $W_{n, d}$.}

Since each of $W_{n, d}$ contains the commutator subgroup $\left[F_{n}, F_{n}\right]$ of $F_{n}$, the IA-automorphism group

$$
\mathrm{IA}_{n}=\left\{\sigma \in \text { Aut } F_{n} \mid x^{-1} x^{\sigma} \in\left[F_{n}, F_{n}\right], x \in F_{n}\right\}
$$

naturally acts on them. Let $\rho=\rho_{n, d}: \mathrm{IA}_{n} \rightarrow$ Aut $W_{n, d}$ be the homomorphism induced from the action. Then we have

Lemma 3.1. For any $n \geq 2$ and $d \geq 2$, the homomorphism $\rho$ is injective.

Proof. For an element $\sigma \in \mathrm{IA}_{n}$, assume $\rho(\sigma)=1$. Since $x_{i}^{\sigma}=x_{i}$ for $2 \leq i \leq n$, in order to see $\sigma=1$, it suffices to show $x_{1}^{\sigma}=x_{1}$.

Set $x_{1}^{\sigma}=x_{1} v$ for some irreducible word $v$. Then we have

$$
x_{1}^{d}=\left(x_{1}^{d}\right)^{\sigma}=\left(x_{1}^{\sigma}\right)^{d}=x_{1} v x_{1} v \cdots x_{1} v,
$$


In the right hand side of the equation above, no cancellation of words happen. In fact, if $v$ is of type $x_{1}^{-1} w$ for some irreducible word $w$, then we have $x_{1}^{d}=w^{d}$. By a usual argument in the Combinatorial group theory, there exists some $z \in F_{n}$ such that $x_{1}=z^{a}$ and $w=z^{b}$ for some $a, b \in \mathbf{Z}$. (See proposition 2.17. in [12].) From the former equation, we have $z=x_{1}$ and $a=1$. Hence

$$
x_{1}^{d} w^{-d}=x_{1}^{d-b d}=1 .
$$

Since $F_{n}$ is torsion free, $b=1$. This shows that $w=x_{1}$. This is a contradiction to the irreducibility of $v=x_{1}^{-1} w$. On the other hand, If $v$ is of type $w x_{1}^{-1}$ for some irreducible word $w$, then we have

$$
x_{1}^{d}=x_{1}^{-1} x_{1}^{d} x_{1}=x_{1}^{-1}\left(x_{1} w^{d} x_{1}^{-1}\right) x_{1}=w^{d} .
$$

By an argument similar to the above, we see that this is also contradiction.

Thus, observing the word length of both side of (4), we obtain that $v=1$. This shows that $\sigma=1$.

By this lemma, we can consider $\mathrm{IA}_{n}$ as a subgroup of each of Aut $W_{n, d}$. In the following, we identify $\mathrm{IA}_{n}$ with the image of it by $\rho$.

Now, let $U_{n, d}$ be the abelianization of $W_{n, d}$. We denote by IA $\left(W_{n, d}\right)$ the IA-automorphism group of $W_{n, d}$. Namely, the group $\operatorname{IA}\left(W_{n, d}\right)$ consists of automorphisms of $W_{n, d}$ which act on $U_{n, d}$ trivially. Let us define an linear order among (3) by

$$
x_{1}^{d}<x_{1} x_{2} x_{1}^{-1}<\ldots<x_{1}^{d-1} x_{2} x_{1}^{-(d-1)}<\ldots<x_{1} x_{n} x_{1}^{-1}<\ldots<x_{1}^{d-1} x_{n} x_{1}^{-(d-1)} .
$$

Then, by a result of Magnus [14] as mentioned in Subsection 2.2, the IA-automorphism group $\mathrm{IA}\left(W_{n, d}\right)$ is finitely generated by automorphisms

$$
\begin{aligned}
& K_{\alpha, \beta}: \alpha \mapsto \alpha^{-1} \beta \alpha, \quad \alpha<\beta, \\
& K_{\alpha, \beta, \gamma}: \alpha \mapsto \alpha[\beta, \gamma], \quad \alpha \neq \beta<\gamma \neq \alpha
\end{aligned}
$$

where $\alpha, \beta, \gamma$ are elements of the basis (3).

On the other hand, from (11), we have

$$
\mathrm{IA}\left(W_{n, d}\right)^{\mathrm{ab}} \cong U_{n, d}^{*} \otimes_{\mathbf{Z}} \Lambda^{2} U_{n, d}
$$

where $U_{n, d}^{*}$ denotes the $\mathbf{Z}$ linear dual group $\operatorname{Hom}_{\mathbf{Z}}\left(U_{n, d}, \mathbf{Z}\right)$ of $U_{n, d}$. This isomorphism is induced from the first Johnson homomorphism

$$
\tau_{1}: \operatorname{IA}\left(W_{n, d}\right) \rightarrow U_{n, d}^{*} \otimes \mathbf{z} \Lambda^{2} U_{n, d} .
$$

In particular, (the coset classes of) the elements $K_{\alpha, \beta} \mathrm{s}$ and $K_{\alpha, \beta, \gamma} \mathrm{s}$ form a basis of $\mathrm{IA}\left(W_{n, d}\right)^{\text {ab }}$ as a free abelian group.

In general, the induced action of $\mathrm{IA}_{n}$ on $U_{n, d}$ by $\rho$ is not trivial. However, its restriction to the kernel

$$
\mathcal{K}_{n}:=\left\{\sigma \in \text { Aut } F_{n} \mid x^{-1} x^{\sigma} \in\left[\left[F_{n}, F_{n}\right],\left[F_{n}, F_{n}\right]\right], \quad x \in F_{n}\right\}
$$

of the Magnus representation is trivial. Namely, $\rho$ induces an embedding

$$
\left.\rho\right|_{\mathcal{K}_{n}}: \mathcal{K}_{n} \hookrightarrow \operatorname{IA}\left(W_{n, d}\right) .
$$

By this embedding, we consider $\mathcal{K}_{n}$ as a subgroup of $\operatorname{IA}\left(W_{n, d}\right)$ for any $d \geq 2$. 


\section{LOWER BOUNDS ON $H_{1}\left(\mathcal{K}_{n}, \mathbf{Z}\right)$}

In the following, we always assume $n \geq 3$ and $d \geq 3$. For each $2 \leq m \leq d-1$, let $\sigma_{m} \in$ Aut $F_{n}$ be an automorphism defined by

$$
x_{i} \mapsto \begin{cases}x_{2}\left[\left[x_{1}, x_{3}\right],\left[x_{1}^{m}, x_{3}\right]\right], & i=2, \\ x_{i}, & i \neq 2 .\end{cases}
$$

Clearly, we see $\sigma_{m} \in \mathcal{K}_{n}$. We show that $\sigma_{2}, \ldots, \sigma_{d-1}$ are linearly independent in $\mathcal{K}_{n}^{\text {ab }}$. To do this, we consider the images of $\sigma_{m}$ by the natural homomorphism

$$
\pi_{n, d}: \mathcal{K}_{n} \hookrightarrow \operatorname{IA}\left(W_{n, d}\right) \rightarrow \operatorname{IA}\left(W_{n, d}\right)^{\text {ab }} .
$$

To begin with, in order to describe $\sigma_{m}$ in $\operatorname{IA}\left(W_{n, d}\right)^{\text {ab }}$ with $K_{\alpha, \beta}$ and $K_{\alpha, \beta, \gamma}$, we consider the action of $\sigma_{m}$ on the basis (3) of $W_{n, d}$. Observing

$$
x_{2}^{\sigma_{m}}=x_{2} \cdot x_{1} x_{3} x_{1}^{-1} \cdot x_{3}^{-1} \cdot x_{1}^{m} x_{3} x_{1}^{-m} \cdot x_{1} x_{3}^{-1} x_{1}^{-1} \cdot x_{3} \cdot x_{1}^{m} x_{3}^{-1} x_{1}^{-m},
$$

we see

$$
\begin{aligned}
&\left(x_{1}^{d}\right)^{\sigma_{m}}=x_{1}^{d}, \\
&\left(x_{1}^{k} x_{i} x_{1}^{-k}\right)^{\sigma_{m}}=x_{1}^{k} x_{i} x_{1}^{-k}, \quad i \neq 2, \\
&\left(x_{1}^{k} x_{2} x_{1}^{-k}\right)^{\sigma_{m}}=x_{1}^{k} x_{2} x_{1}^{-k} \cdot x_{1}^{k+1} x_{3} x_{1}^{-(k+1)} \cdot x_{1}^{k} x_{3}^{-1} x_{1}^{-k} \cdot x_{1}^{m+k} x_{3} x_{1}^{-(m+k)} \\
& \cdot x_{1}^{k+1} x_{3}^{-1} x_{1}^{-(k+1)} \cdot x_{1}^{k} x_{3} x_{1}^{-k} \cdot x_{1}^{m+k} x_{3}^{-1} x_{1}^{-(m+k)} .
\end{aligned}
$$

Hence, for any $0 \leq k \leq d-1$, if we denote by $\omega_{m, k}$ the automorphism of $W_{n, d}$ defined by

$$
\begin{gathered}
x_{1}^{k} x_{2} x_{1}^{-k} \mapsto x_{1}^{k} x_{2} x_{1}^{-k} \cdot x_{1}^{k+1} x_{3} x_{1}^{-(k+1)} \cdot x_{1}^{k} x_{3}^{-1} x_{1}^{-k} \cdot x_{1}^{m+k} x_{3} x_{1}^{-(m+k)} \\
\cdot x_{1}^{k+1} x_{3}^{-1} x_{1}^{-(k+1)} \cdot x_{1}^{k} x_{3} x_{1}^{-k} \cdot x_{1}^{m+k} x_{3}^{-1} x_{1}^{-(m+k)},
\end{gathered}
$$

then

$$
\sigma_{m}=\omega_{m, 0} \omega_{m, 2} \cdots \omega_{m, d-1} .
$$

Here we remark that for any $0 \leq k, l \leq d-1$, the automorphisms $\omega_{m, k}$ and $\omega_{m, l}$ are commutative in Aut $W_{n, d}$.

Let us describe $\omega_{m, k}$ in $\operatorname{IA}\left(W_{n, d}\right)^{\text {ab }}$ with $K_{\alpha, \beta}$ and $K_{\alpha, \beta, \gamma}$ for each $0 \leq k \leq d-1$.

Case 1. The case of $0 \leq k \leq d-1-m$.

In this case, we have $k+1 \leq d-1$ and $m+k \leq d-1$. Thus, by (6) and Lemma 2.1, we see

$$
\begin{aligned}
\omega_{m, k}= & K_{x_{1}^{k} x_{2} x_{1}^{-k}, x_{1}^{k} x_{3} x_{1}^{-k}} K_{x_{1}^{k} x_{2} x_{1}^{-k}, x_{1}^{m+k} x_{3} x_{1}^{-(m+k)}, x_{1}^{k} x_{3} x_{1}^{-k}} \\
& \cdot K_{x_{1}^{k} x_{2} x_{1}^{-k}, x_{1}^{k+1} x_{3} x_{1}^{-(k+1)}, x_{1}^{m+k} x_{3} x_{1}^{-(m+k)}} \\
& \cdot K_{x_{1}^{k} x_{2} x_{1}^{-k}, x_{1}^{k} x_{3} x_{1}^{-k}, x_{1}^{k+1} x_{3} x_{1}^{-(k+1)} K_{x_{1}^{k} x_{2} x_{1}^{-k}, x_{1}^{k} x_{3} x_{1}^{-k}}}
\end{aligned}
$$

in $\operatorname{IA}\left(W_{n, d}\right)$, and hence,

$$
\begin{aligned}
\omega_{m, k}= & K_{x_{1}^{k} x_{2} x_{1}^{-k}, x_{1}^{m+k} x_{3} x_{1}^{-(m+k)}, x_{1}^{k} x_{3} x_{1}^{-k}}+K_{x_{1}^{k} x_{2} x_{1}^{-k}, x_{1}^{k+1} x_{3} x_{1}^{-(k+1)}, x_{1}^{m+k} x_{3} x_{1}^{-(m+k)}} \\
& +K_{x_{1}^{k} x_{2} x_{1}^{-k}, x_{1}^{k} x_{3} x_{1}^{-k}, x_{1}^{k+1} x_{3} x_{1}^{-(k+1)} .}
\end{aligned}
$$

in $\operatorname{IA}\left(W_{n, d}\right)^{\mathrm{ab}}$. 
Case 2. The case of $d-m \leq k \leq d-2$.

In this case, we have $k+1 \leq d-1<m+k$. Hence (6) is written as

$$
\begin{gathered}
x_{1}^{k} x_{2} x_{1}^{-k} \mapsto x_{1}^{k} x_{2} x_{1}^{-k} \cdot x_{1}^{k+1} x_{3} x_{1}^{-(k+1)} \cdot x_{1}^{k} x_{3}^{-1} x_{1}^{-k} \cdot x_{1}^{d} \cdot x_{1}^{m+k-d} x_{3} x_{1}^{-(m+k-d)} \cdot x_{1}^{-d} \\
\cdot x_{1}^{k+1} x_{3}^{-1} x_{1}^{-(k+1)} \cdot x_{1}^{k} x_{3} x_{1}^{-k} \cdot x_{1}^{d} \cdot x_{1}^{m+k-d} x_{3}^{-1} x_{1}^{-(m+k-d)} \cdot x_{1}^{-d} .
\end{gathered}
$$

Then using Lemma 2.2, we obtain

$$
\begin{aligned}
\omega_{m, k}= & K_{x_{1}^{k} x_{2} x_{1}^{-k}, x_{1}^{m+k-d} x_{3} x_{1}^{-(m+k-d)}, x_{1}^{k} x_{3} x_{1}^{-k}}+K_{x_{1}^{k} x_{2} x_{1}^{-k}, x_{1}^{k+1} x_{3} x_{1}^{-(k+1)}, x_{1}^{m+k-d} x_{3} x_{1}^{-(m+k-d)}} \\
& +K_{x_{1}^{k} x_{2} x_{1}^{-k}, x_{1}^{k} x_{3} x_{1}^{-k}, x_{1}^{k+1} x_{3} x_{1}^{-(k+1)} .}
\end{aligned}
$$

in $\operatorname{IA}\left(W_{n, d}\right)^{\mathrm{ab}}$.

Case 3. The case of $k=d-1$.

In this case, we have $k+1=d$ and $d \leq m+k$. Hence (6) is written as

$$
\begin{aligned}
x_{1}^{d-1} x_{2} x_{1}^{-(d-1)} \mapsto x_{1}^{d-1} & x_{2} x_{1}^{-(d-1)} \\
& \cdot x_{1}^{d} \cdot x_{3} \cdot x_{1}^{-d} \cdot x_{1}^{d-1} x_{3}^{-1} x_{1}^{-(d-1)} \cdot x_{1}^{d} \cdot x_{1}^{m-1} x_{3} x_{1}^{-(m-1)} \cdot x_{1}^{-d} \\
& \cdot x_{1}^{d} \cdot x_{3}^{-1} \cdot x_{1}^{-d} \cdot x_{1}^{d-1} x_{3} x_{1}^{-(d-1)} \cdot x_{1}^{d} \cdot x_{1}^{m-1} x_{3}^{-1} x_{1}^{-(m-1)} \cdot x_{1}^{-d} .
\end{aligned}
$$

Then using Lemma 2.3, we obtain

$$
\begin{aligned}
\omega_{m, d-1}= & K_{x_{1}^{d-1} x_{2} x_{1}^{-(d-1)}, x_{1}^{m-1} x_{3} x_{1}^{-(m-1)}, x_{1}^{d-1} x_{3} x_{1}^{-(d-1)}}+K_{x_{1}^{d-1} x_{2} x_{1}^{-(d-1)}, x_{3}, x_{1}^{m-1} x_{3} x_{1}^{-(m-1)}} \\
& +K_{x_{1}^{d-1} x_{2} x_{1}^{-(d-1)}, x_{1}^{d-1} x_{3} x_{1}^{-(d-1)}, x_{3}} .
\end{aligned}
$$

in $\operatorname{IA}\left(W_{n, d}\right)^{\mathrm{ab}}$.

Therefore for $2 \leq m \leq d-1$, we obtain

$$
\begin{aligned}
& \sigma_{m}=\sum_{0 \leq k \leq d-1-m}\left\{K_{x_{1}^{k} x_{2} x_{1}^{-k}, x_{1}^{m+k} x_{3} x_{1}^{-(m+k)}, x_{1}^{k} x_{3} x_{1}^{-k}}\right. \\
& \left.+K_{x_{1}^{k} x_{2} x_{1}^{-k}, x_{1}^{k+1} x_{3} x_{1}^{-(k+1)}, x_{1}^{m+k} x_{3} x_{1}^{-(m+k)}}+K_{x_{1}^{k} x_{2} x_{1}^{-k}, x_{1}^{k} x_{3} x_{1}^{-k}, x_{1}^{k+1} x_{3} x_{1}^{-(k+1)}}\right\} \\
& +\sum_{d-m \leq k \leq d-1}\left\{K_{x_{1}^{k} x_{2} x_{1}^{-k}, x_{1}^{m+k-d} x_{3} x_{1}^{-(m+k-d)}, x_{1}^{k} x_{3} x_{1}^{-k}}\right. \\
& \left.+K_{x_{1}^{k} x_{2} x_{1}^{-k}, x_{1}^{k+1} x_{3} x_{1}^{-(k+1)}, x_{1}^{m+k-d} x_{3} x_{1}^{-(m+k-d)}}+K_{x_{1}^{k} x_{2} x_{1}^{-k}, x_{1}^{k} x_{3} x_{1}^{-k}, x_{1}^{k+1} x_{3} x_{1}^{-(k+1)}}\right\}
\end{aligned}
$$

in $\operatorname{IA}\left(W_{n, d}\right)^{\mathrm{ab}}$. Using this, we obtain

Lemma 4.1. For any $n \geq 3$ and $d \geq 3$, the elements $\sigma_{2}, \ldots, \sigma_{d-1}$ are linearly independent in $\operatorname{IA}\left(W_{n, d}\right)^{\mathrm{ab}}$.

Proof. Suppose

$$
a_{2} \sigma_{2}+\cdots+a_{d} \sigma_{d}=0 \in \operatorname{IA}\left(W_{n, d}\right)^{\mathrm{ab}} .
$$

Then, observing the coefficients of $K_{x_{2}, *, *}$ in the left hand side of the equation above, we see

$$
\sum_{2 \leq m \leq d-1} a_{m}\left\{K_{x_{2}, x_{1}^{m} x_{3} x_{1}^{-m}, x_{3}}+K_{x_{2}, x_{1} x_{3} x_{1}^{-1}, x_{1}^{m} x_{3} x_{1}^{-m}}+K_{x_{2}, x_{3}, x_{1} x_{3} x_{1}^{-1}}\right\}=0 .
$$

Hence, we have $a_{2}=\cdots=a_{d-1}=0$.

This Lemma induces our main theorem. 
Theorem 4.1. For $n \geq 3, \mathcal{K}_{n}^{\text {ab }}$ is not finitely generated.

Proof. For any $d \geq 3$, the image of the induced map

$$
\mathcal{K}_{n}^{\mathrm{ab}} \rightarrow \operatorname{IA}\left(W_{n, d}\right)^{\mathrm{ab}}
$$

from the natural homomorphism $\pi_{n, d}: \mathcal{K}_{n} \rightarrow \mathrm{IA}\left(W_{n, d}\right)^{\text {ab }}$ contains a free abelian group of rank $d-2$. Since we can take $d \geq 3$ arbitrarily, we obtain the required result. This completes the proof of Theorem 4.1 ,

As a Corollary, we have

Corollary 4.1. For $n \geq 3, \mathcal{K}_{n}$ is not finitely generated.

\section{The CASE WHERE $n=2$}

In this section, we show that $\mathcal{K}_{2}$ is not finitely generated. More precisely, we show that $\mathcal{K}_{2}$ is isomorphic to the second derived subgroup $\left[\left[F_{2}, F_{2}\right],\left[F_{2}, F_{2}\right]\right]$ of $F_{2}$.

To begin with, we remark that $\mathrm{IA}_{2}$ is equal to the inner automorphism group $\operatorname{Inn} F_{2}$ of $F_{2}$ due to Nielsen [19]. Furthermore, Bachmuth [1] showed that $\mathrm{IA}_{2}^{M}$ coincides with Inn $F_{2}^{M}$. Hence we see

$$
\mathcal{K}_{2}=\operatorname{Ker}\left(\operatorname{Inn} F_{2} \stackrel{\mu_{2}}{\longrightarrow} \operatorname{Inn} F_{2}^{M}\right) .
$$

In general, for any group $G$, a homomorphism from $G$ onto the inner automorphism group Inn $G$ of $G$ defined by

$$
g \mapsto \iota_{g}=\left(x \mapsto g^{-1} x g, \quad x \in G\right)
$$

induces an isomorphism $\operatorname{Inn} G \cong G / Z(G)$ where $Z(G)$ denotes the center of $G$. Since the center of $F_{2}$ is trivial, we have $\operatorname{Inn} F_{2} \cong F_{2}$.

Here we show that $Z\left(F_{n}^{M}\right)$ is trivial for any $n \geq 2$. By a result of Magnus [13], $F_{n}^{M}$ is faithfully represented in the group of $2 \times 2$ matrices of the form

$$
\left(\begin{array}{ll}
x & L \\
0 & 1
\end{array}\right)
$$

where $x \in H$ and $L$ is a linear form $v_{1} t_{1}+\cdots+v_{n} t_{n}$ in the indeterminates $t_{1}, \ldots, t_{n}$ with coefficients in $\mathbf{Z}[H]$. Here we identify $F_{n}^{M}$ with the image of it by the representation. The image of $x_{i} \in F_{n}^{M}$ by this representation is given by

$$
\left(\begin{array}{cc}
x_{i} & t_{i} \\
0 & 1
\end{array}\right) .
$$

Let

$$
\left(\begin{array}{cc}
x & v_{1} t_{1}+\cdots+v_{n} t_{n} \\
0 & 1
\end{array}\right) \in Z\left(F_{n}^{M}\right) .
$$

Then for any $1 \leq i \leq n$, we have

$$
\begin{aligned}
& \left(\begin{array}{cc}
x & v_{1} t_{1}+\cdots+v_{n} t_{n} \\
0 & 1
\end{array}\right)\left(\begin{array}{cc}
x_{i} & t_{i} \\
0 & 1
\end{array}\right)=\left(\begin{array}{cc}
x x_{i} & v_{1} t_{1}+\cdots+\left(v_{i}+x\right) t_{i}+\cdots+v_{n} t_{n} \\
0 & 1
\end{array}\right), \\
& \left(\begin{array}{cc}
x_{i} & t_{i} \\
0 & 1
\end{array}\right)\left(\begin{array}{cc}
x & v_{1} t_{1}+\cdots+v_{n} t_{n} \\
0 & 1
\end{array}\right)=\left(\begin{array}{cc}
x_{i} x & \left(x_{i} v_{1}\right) t_{1}+\cdots+\left(x_{i} v_{i}+1\right) t_{i}+\cdots+\left(x_{i} v_{n}\right) t_{n} \\
0 & 1
\end{array}\right),
\end{aligned}
$$


and hence,

$$
\begin{aligned}
x_{i} v_{j} & =v_{j}, \quad 1 \leq j \leq n, \quad j \neq i, \\
v_{i}+x & =x_{i} v_{i}+1 .
\end{aligned}
$$

Since $\mathbf{Z}[H]$ is a domain, we see $v_{j}=0$ for $j \neq i$ from the former equation above. Since we can take $1 \leq i \leq n$ arbitrarily, we obtain $v_{1}=\cdots=v_{n}=0 \in \mathbf{Z}[H]$. Then $x=1 \in H$. Therefore we see $Z\left(F_{n}^{M}\right)$ is trivial.

By the argument above, we verify that $\operatorname{Inn} F_{2}^{M} \cong F_{2}^{M}$, and that $\mathcal{K}_{2}$ is the kernel of the natural projection $F_{2} \rightarrow F_{2}^{M}$. That is,

Proposition 5.1. $\mathcal{K}_{2}=\left[\left[F_{2}, F_{2}\right],\left[F_{2}, F_{2}\right]\right]$.

By an easy argument in the Combinatorial group theory, we see that $\left[\left[F_{2}, F_{2}\right],\left[F_{2}, F_{2}\right]\right]$ is a free group of infinite rank as follows. First, we consider

Lemma 5.1. If $F$ is a free group of countably infinite rank, so is its commutator subgroup $[F, F]$.

Proof. Let $Y:=\left\{y_{1}, y_{2}, \ldots\right\}$ be the set of generators of $F$. Then, applying the Reidemeister-Schreier method to $Y$ and a Schreier-transversal

$$
S:=\left\{y_{1}^{e_{1}} y_{2}^{e_{2}} \cdots \mid e_{i} \in \mathbf{Z}, \text { all but finite } e_{i} \text { s are } 0 .\right\}
$$

of $[F, F]$ of $F$, we see that $[F, F]$ is a free group with basis

$$
\left\{(s, y)=s y(\overline{s y})^{-1} \mid s \in S, y \in Y, \quad(s, y) \neq 1\right\} .
$$

Hence, it suffices to show the set above contains infinitely many elements.

Let us consider $y=y_{1}$ and $s=y_{1}^{e_{1}} y_{2}$ for any $\mathbf{Z}$. Then we have

$$
(s, y)=y_{1}^{e_{1}} y_{2} y_{1} y_{2}^{-1} y_{1}^{-\left(e_{1}+1\right)} \neq 1
$$

and

$$
y_{1}^{e_{1}} y_{2} y_{1} y_{2}^{-1} y_{1}^{-\left(e_{1}+1\right)} \neq y_{1}^{e_{1}^{\prime}} y_{2} y_{1} y_{2}^{-1} y_{1}^{-\left(e_{1}^{\prime}+1\right)}
$$

if $e_{1} \neq e_{1}^{\prime}$. This completes the proof of Lemma 5.1,

In general, since the commutator subgroup $\left[F_{n}, F_{n}\right]$ of the free group $F_{n}$ is a free group of countably infinite rank for $n \geq 2$, using Lemma 5.1, we conclude

Proposition 5.2. The group $\mathcal{K}_{2}$ is a free group of countably infinite rank for $n \geq 2$.

\section{ACKnowledgments}

This research is supported by JSPS Research Fellowship for Young Scientists and the Global COE program at Kyoto University.

\section{REFERENCES}

[1] S. Bachmuth; Automorphisms of free metabelian groups, Trans. Amer. Math. Soc. 118 (1965), 93-104.

[2] S. Bachmuth and H. Y. Mochizuki; The non-finite generation of $\operatorname{Aut}(G), G$ free metabelian of rank 3, Trans. Amer. Math. Soc. 270 (1982), 693-700.

[3] S. Bachmuth and H. Y. Mochizuki; $\operatorname{Aut}(F) \rightarrow \operatorname{Aut}\left(F / F^{\prime \prime}\right)$ is surjective for free group for rank $\geq 4$, Trans. Amer. Math. Soc. 292, no. 1 (1985), 81-101. 
[4] J. S. Birman; Braids, Links, and Mapping Class Groups, Annals of Math. Studies 82, Princeton University Press (1974).

[5] F. Cohen and J. Pakianathan; On Automorphism Groups of Free Groups, and Their Nilpotent Quotients, preprint.

[6] F. Cohen and J. Pakianathan; On subgroups of the automorphism group of a free group and associated graded Lie algebras, preprint.

[7] T. Church and B. Farb; Infinite generation of the kernels of the Magnus and Burau representations, preprint, arXiv:math.GR/0909.4825.

[8] B. Farb; Automorphisms of $F_{n}$ which act trivially on homology, in preparation.

[9] R. Fox; Free differential calculus I, Ann. of Math. 57 (1953), 547-560.

[10] N. Kawazumi, Cohomological aspects of Magnus expansions, preprint, arXiv:math.GT/0505497.

[11] S. Krstić, J. McCool; The non-finite presentability in $I A\left(F_{3}\right)$ and $G L_{2}\left(\mathbf{Z}\left[t, t^{-1}\right]\right)$, Invent. Math. 129 (1997), 595-606.

[12] R. C. Lyndon and P. E. Schupp; Combinatorial Group Theory, Springer-Verlag (1977).

[13] W. Magnus; On a theorem of Marshall Hall, Ann. of Math. 40 (1930), 764-768.

[14] W. Magnus; Über n-dimensinale Gittertransformationen, Acta Math. 64 (1935), 353-367.

[15] W. Magnus and A. Peluso; On a theorem of V. I. Arnold, Com. on Pure and App. Math., XXII (1969), 683-692.

[16] S. Morita, Abelian quotients of subgroups of the mapping class group of surfaces, Duke Mathematical Journal 70 (1993), 699-726.

[17] S. Morita, Structure of the mapping class groups of surfaces: a survey and a prospect, Geometry and Topology Monographs Vol. 2 (1999), 349-406.

[18] S. Morita, Cohomological structure of the mapping class group and beyond, Proc. of Symposia in Pure Math. 74 (2006), 329-354.

[19] J. Nielsen; Die Isomorphismen der allgemeinen unendlichen Gruppe mit zwei Erzeugenden, Math. Ann. 78 (1918), 385-397.

[20] T. Satoh; New obstructions for the surjectivity of the Johnson homomorphism of the automorphism group of a free group, Journal of the London Mathematical Society, (2) 74 (2006) 341-360.

[21] T. Satoh; The cokernel of the Johnson homomorphisms of the automorphism group of a free metabelian group, Transactions of American Mathematical Society, 361 (2009), 2085-2107.

[22] M. Suzuki; The Magnus representation of the Torelli group $\mathcal{I}_{g, 1}$ is not faithful for $g \geq 2$, Proc. Amer. Math. Soc. 130 (2002), 909-914.

Department of Mathematics, Graduate School of Science, Kyoto University, KiTASHIRAKaWAOIWAKe CHO, SAKYO-KU, KYOto CITY 606-8502, JAPAN

E-mail address: takao@math.kyoto-u.ac.jp 